\title{
Anatomical Tenodesis Reconstruction Using Free Split Peroneal Brevis Tendon for Severe Chronic Lateral Ankle Instability
}

\author{
Takeshi Hashimoto and Tetsuro Kokubo \\ Sports Medicine Research Center, Keio University, Yokohama, Japan \\ (Received for publication on August 31, 2021) \\ (Revised for publication on October 6, 2021) \\ (Accepted for publication on October 11, 2021) \\ (Published online in advance on November 10, 2021)
}

\begin{abstract}
Many operative procedures have been reported for the management of chronic lateral ankle instability, and anatomical reconstructions are an excellent option. However, if the remnants of the ligaments are considerably damaged, anatomical reconstructions using such remnants can be difficult. In cases such as these, tenodesis stabilization may be required. However, tenodesis stabilization often restricts the range of ankle movement. The purpose of this study was to determine the effectiveness of a new procedure that we developed to mitigate the problems associated with tenodesis stabilization procedures. We installed grafts in the original anatomical position by devising a system for positioning the drill holes in the bones so that our procedure did not restrict the range of ankle movement. A retrospective review of 37 patients (13 men, 24 women) with a mean age of 30.2 (range, 16-66) years was performed at an average of 69 (range, 47-77) months after the surgery. The average American Orthopaedic Foot and Ankle Society ankle-hindfoot score improved significantly from 65.6 (range, 47-77) points preoperatively to 98.0 (range, $87-100)$ points postoperatively $(P<\mathbf{0 . 0 0 1})$. With the number of subjects available, no significant differences were detected between the postoperative mean ranges of movement of the ankle and subtalar joints and those of the preoperative ankle. Patients who underwent anatomical tenodesis reconstructions with a free split peroneal brevis tendon showed good outcomes after a 69-month followup period. (DOI: 10.2302/kjm.2021-0014-OA)
\end{abstract}

Keywords: chronic ankle instability, reconstruction, ligament, ankle joint, tenodesis stabilization

\section{Introduction}

Ankle sprains are one of the most common orthopedic injuries. Even if ankle sprains are treated appropriately, chronic lateral ankle instability may occur in $10 \%$ to $20 \%$ of patients. Furthermore, such instability can lead to osteoarthritis of the ankle. ${ }^{1}$ Patients with chronic lateral ankle instability are best managed with a brace and rehabilitation. However, patients with persistent pain and ankle instability after conservative treatment may be candidates for operative repair of the lateral ankle ligaments.

Many operative procedures have been reported for the management of chronic lateral ankle instability. These procedures are classified into two general categories: (1) anatomical reconstructions, i.e., procedures that repair the ligaments directly from remnants, with or without augmentation, ${ }^{2-8}$ and (2) tenodesis stabilizations, i.e., procedures that reconstruct the ligaments using a free or a split tendon graft. ${ }^{9-13}$ Anatomical reconstructions are an excellent option because they are simple to perform and complications are rare. However, if the remnants of the ligaments are severely damaged, anatomical reconstruction using the remnants is not easy to perform, and tenodesis stabilization may be needed.

Several clinical and biomechanical studies have shown that tenodesis stabilization often restricts the range of motion (ROM) of the ankle and is also associated with nerve injuries. The purpose of the current study was to 
Table 1. Demographic data of patients

\begin{tabular}{lc} 
Parameter & Value \\
\hline Age at time of operation* (years) & $30.2 \pm 11.9(16-66)$ \\
Sex (male/female) & $13 / 24$ \\
Affected ankle** (right/left) & $23 / 16$ \\
Tegner activity scale* & $5.2 \pm 1.9(1-9)$ \\
Stress X-ray findings & $7.8 \pm 2.2(4-14)$ \\
Anterior drawer distance* (mm) & $15.8 \pm 3.3(10-23)$ \\
Talar tilt angle* $\left({ }^{\circ}\right)$ & \\
\hline *Values given as the mean \pm SD with range in parentheses. & \\
**Two patients had bilateral involvement. &
\end{tabular}

determine the efficacy of a new procedure that we developed to overcome the problems associated with tenodesis stabilization procedures for the treatment of chronic lateral ankle instability in patients with severely damaged ligaments. Our hypothesis was that the new tenodesis stabilization procedure would enable good outcomes with no restrictions of the ankle ROM.

\section{Materials and Methods}

\section{Ethical approval}

All procedures were carried out in accordance with the ethical standards of the responsible committee at the institution at which the studies were conducted (IRB approval number 2013-04) and with the 1964 Helsinki declaration and its later amendments or comparable ethical standards. Informed consent was obtained from all participants included in the study.

\section{Patients}

Between 1998 and 2009, a total of 48 lateral ankle ligament reconstruction procedures were performed in 46 patients, with two patients undergoing a bilateral procedure. Of these 46 patients, 37 were available for clinical assessment that included a physical examination, an interview, and a review of medical records. The remaining nine patients were not able or were unwilling to visit the hospital for clinical re-evaluation. The subjects were 13 men and 24 women with a mean age of 30.2 years (range, $16-66$ years). Of the 39 procedures included in this study, 23 were performed on right ankles and 16 on left ankles. Two patients underwent bilateral procedures. The mean Tegner activity scale score was 5.2 (range, 1-9) (Table 1). If radiographs suggested osteochondral dissecans or any minor fracture, magnetic resonance imaging or computed tomography was performed to determine whether the patients should be excluded from the study.

The lateral ankle ligament reconstruction procedure was indicated when there was confirmation of lateral ankle instability associated with pain for a duration of greater than 6 months with evidence of ankle instability upon stress radiograph examination. It was assumed that the patient had ankle instability if the anterior drawer distance was $8 \mathrm{~mm}$ or greater and/or the talar tilt angle was $15^{\circ}$ or greater. Before surgery, all the patients were treated with physical therapy, ankle strengthening, proprioceptive training, and bracing. The patients were referred to a single orthopedic surgeon (senior author) for surgical management.

\section{Surgical procedure}

The operation was performed with patient under general anesthesia. The patients were placed in the decubitus position, and a thigh tourniquet was applied. A curvilinear 10-cm-long skin incision was made over the lateral malleolus, and then the anterior talofibular ligament and the calcaneofibular ligaments were exposed. The sural nerve was identified in the posterior region of the lateral malleolus and carefully exposed to the fifth metatarsal bone; the nerve was then marked with an elastic band to protect it. The peroneal muscle fascia was incised, and the peroneus brevis tendon was isolated. The anterior half of the tendon was harvested from the musculotendinous junction to the distal end of the base of the fifth metatarsal bone. The harvested tendon was typically $12 \mathrm{~cm}$ in length from female patients and $15 \mathrm{~cm}$ in length from male patients. The grafts were created so that, as far as possible, they were of the same diameter throughout their length.

Four bone tunnels were then made at the insertion points of the anterior talofibular ligaments and calcaneofibular ligaments using a drill with a diameter of $3.5 \mathrm{~mm}$. This was done in a manner such that the grafts simulated the routes of physiological ligaments. The bone tunnels were created as follows: V-shaped bone tunnels were made using the drill at an angle of $45^{\circ}$ to the bone surface at the physiological insertion point of the anterior talofibular and calcaneofibular ligaments. Then, two bone tunnels were also made at the lateral malleolus using the same drill. The tunnels were widened using curved Pean forceps that could be smoothly removed after widen- 


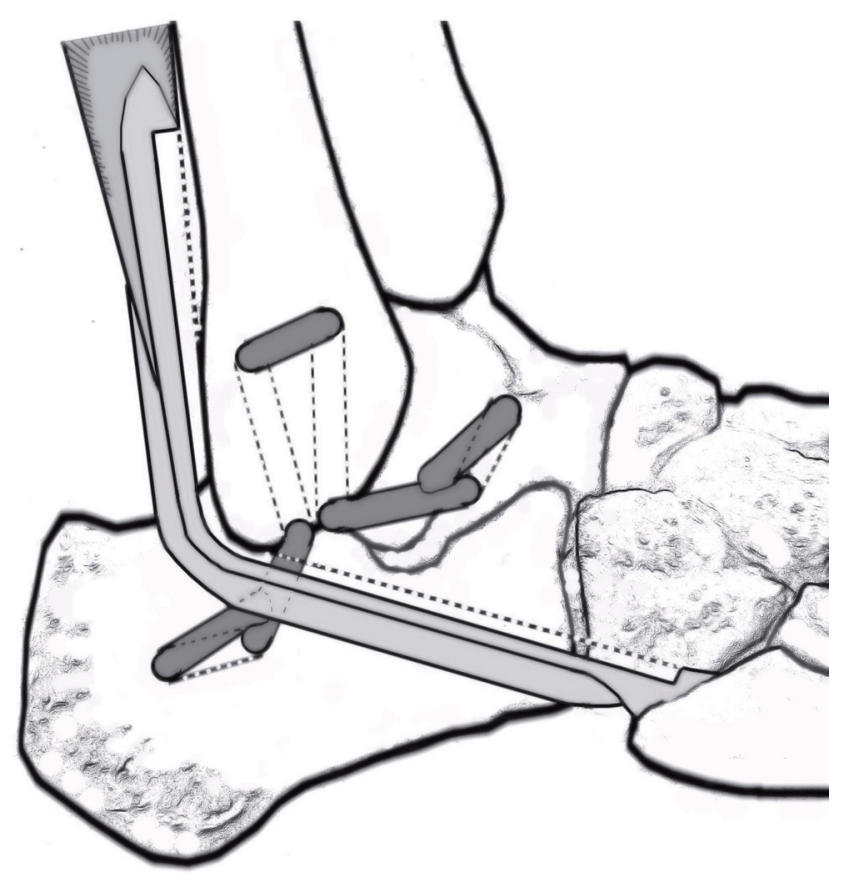

Fig. 1 Sagittal view showing the reconstruction of the lateral ligaments. Dark gray areas: harvested peroneal brevis tendon; dashed lines, bone tunnels; light gray areas, peroneal brevis and longus tendons; dashed line located above peroneal brevis tendon, section harvested for use as a graft.

ing was complete. Using Deschamps needles, $2-0$ nylon threads were passed through the bone tunnels. The grafts were then passed through the bone tunnels using the 2-0 nylon threads as guide threads (Fig. 1, 2).

The grafts were then tightened and sutured at the insertion points of the ligaments using 2-0 non-absorbable sutures with the ankle in the neutral position. The anterior talofibular ligaments were then strongly tightened with as much tension as possible. The calcaneofibular ligaments were tightened with $90 \%$ of the tension of the anterior talofibular ligaments. The remnant ligaments in 27 of the 37 patients were then cut and sutured to the reconstructed ligaments.

Postoperatively, the ankle was immobilized in the neutral position using a short leg cast for 4 weeks. Active ROM exercises of the toes were commenced in the immediate postoperative period. Partial weight bearing was allowed at 1 week postoperatively. At 4 weeks postoperatively, the cast was removed and an ankle brace was applied. Patients were then encouraged to begin active dorsiflexion and plantar flexion and were upgraded to full weight bearing. Proprioception was retrained using a wobble board and ankle strengthening exercises were also initiated 4 weeks postoperatively. Patients were en-

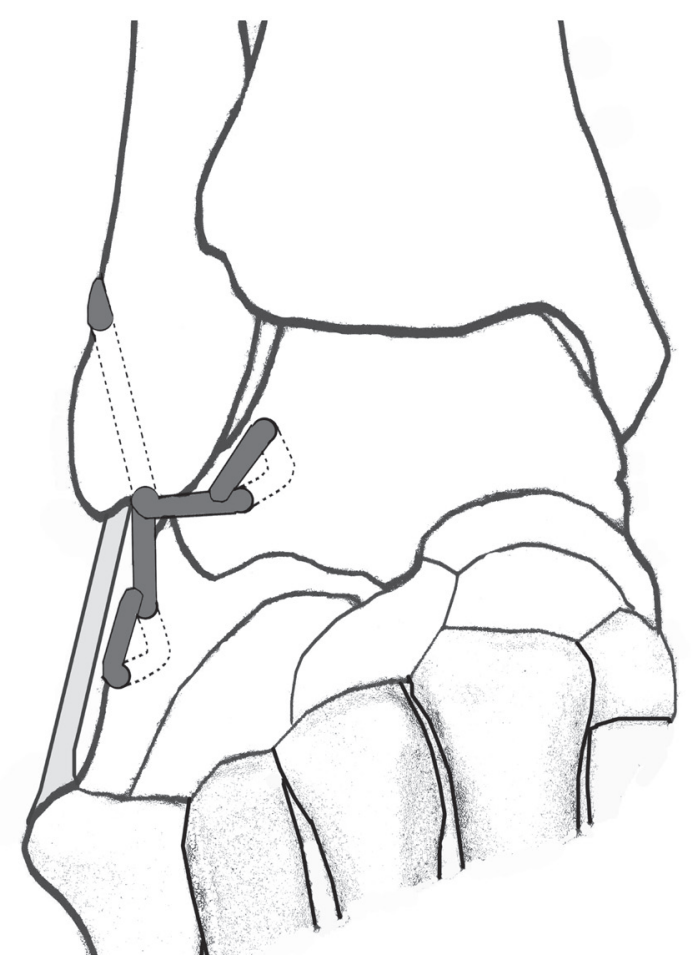

Fig. 2 Coronal view showing the reconstruction of the lateral ligaments. Dark gray areas, harvested peroneal brevis tendon; dashed lines, bone tunnels; light gray area, peroneal brevis and longus tendons.

couraged to achieve their previous level of activity at 8 weeks postoperatively. Finally, they were allowed to return to sporting activities at 4 months postoperatively.

\section{Clinical and radiographic evaluation}

Patients were examined on the day before surgery. The clinical condition of the ankle was graded according to the rating system of the American Orthopaedic Foot and Ankle Society (AOFAS) Ankle-Hindfoot scale. ${ }^{14}$ Postoperative evaluation of the patients included a direct physical examination, a review of the medical records, and an interview with a questionnaire completed postoperatively. The AOFAS Ankle-Hindfoot scale was also used for clinical evaluation at the postoperative examination. The results were graded as excellent (100 to 91 points), good (90 to 81 points), fair ( 80 to 71 points), or poor (less than 70 points), as described by Becker et al. ${ }^{15}$ During the final examination, the ankle ROM (dorsiflexion and plantar flexion) and subtalar ROM (inversion and eversion) were assessed.

Each patient was evaluated pre- and postoperatively using stress radiography (Telos, Hungen, Germany) under a $150-\mathrm{N}$ force without anesthesia. The anterior drawer 
Table 2. Preoperative and postoperative clinical and radiographic evaluations

\begin{tabular}{lccc}
\hline \hline Parameter & Preoperative & Postoperative & $* P$ value \\
\hline AOFAS Ankle-Hindfoot score & $65.6 \pm 4.8(47-77)$ & $98.0 \pm 4.1(87-100)$ & $P<0.05$ \\
Range of movement of dorsiflexion $\left(^{\circ}\right)$ & $16.0 \pm 3.7(10-23)$ & $15.3 \pm 3.8(8-22)$ & $P=0.10$ \\
Range of movement of plantar flexion $\left(^{\circ}\right)$ & $59.4 \pm 6.9(47-75)$ & $58.5 \pm 7.3(46-74)$ & $P=0.24$ \\
Range of movement of inversion $\left(^{\circ}\right)$ & $21.4 \pm 4.7(12-30)$ & $21.2 \pm 4.7(11-30)$ & $P=0.81$ \\
Range of movement of eversion $\left(^{\circ}\right)$ & $8.4 \pm 2.0(6-14)$ & $8.0 \pm 2.0(6-14)$ & $P=0.08$ \\
Tegner activity scale & $5.2 \pm 1.9(1-9)$ & $5.2 \pm 1.9(1-9)$ & $P=0.57$ \\
Stress X-ray findings & & & \\
Anterior drawer distance (mm) & $7.8 \pm 2.2(4-14)$ & $4.0 \pm 1.4(1-7)$ & $P<0.001$ \\
Talar tilt angle $\left(^{\circ}\right)$ & $15.8 \pm 3.3(10-23)$ & $6.6 \pm 1.5(3-10)$ & $P<0.001$ \\
\hline
\end{tabular}

Values are given as the mean \pm SD with the range in parentheses.

*Pre- and postoperative values were compared.

distance was defined as the anterior displacement of the talus in the ankle mortise with a posterior-to-anterior applied load on the foot using lateral radiographs. Clinical and radiological examinations were performed postoperatively on the last day of follow-up.

\section{Statistical analysis}

The sample size was calculated to detect a mean difference in the AOFAS score of 10 with a standard deviation (SD) of 4.8. The error was set at 0.05 and the power level at $90 \%$. The required sample size was a minimum of 25 patients. Differences between groups were compared using a two-tailed Student t-test for normally distributed values. The results are presented as the mean and range. The level of significance was set at $P<0.05$. Statistical analyses were performed using SPSS software (version 22; IBM Corp., Armonk, NY, USA).

\section{Results}

The mean follow-up period was 69 (range, 24-153) months after the operation. Operations were performed on average 8.6 (range, 6-30) months after the start of conservative treatment.

The mean AOFAS Ankle-Hindfoot score improved significantly from 65.6 (range, 47-77) points preoperatively to 98.0 (range, $87-100)$ points postoperatively $(P$ $<0.05)$. No significant difference in the mean Tegner activity scale was seen between preoperative (5.2; range, 1-9 points) and postoperative (5.2; range, 1-9 points) measurements. No significant differences between the preoperative and postoperative mean ROM of the ankle (plantar flexion and dorsiflexion) or subtalar (inversion and eversion) joints of the affected ankle were found. The AOFAS Ankle-Hindfoot scale results were classified as excellent in 31 patients (84\%) and good in six patients $(16 \%)$

Before the injury, 9 of the 37 patients had participated in sports. Seven of these nine patients were able to return to their pre-injury levels of physical activity at 4-12 months after the operation. The remaining two patients did not return to sports because of social problems.

The mean anterior drawer distance improved significantly from 7.8 (range, 4-14) $\mathrm{mm}$ preoperatively to 4.0 (range, 1-7) $\mathrm{mm}$ postoperatively. The mean talar tilt angle improved significantly from $15.8^{\circ}$ (range, $10^{\circ}-23^{\circ}$ ) preoperatively to $6.6^{\circ}$ (range, $3^{\circ}-10^{\circ}$ ) degrees postoperatively.

An early superficial infection that required the administration of intravenous antibiotics developed in one patient; this patient later resumed a normal postoperative course. A 28-year-old male patient developed irritation and numbness in the sural nerve region on the lateral side of the foot with an associated AOFAS Ankle-Hindfoot score of 90 . One patient developed stiffness of the ankle joint as a result of a decreased inversion ROM. No major complications, such as repeat rupture or deep tissue infection, occurred in this series. The overall complication rate was $8.1 \%$ ( $3 / 37$ patients). The improvements in the ankle scores are summarized in Table 2.

\section{Discussion}

Many surgical techniques for the management of chronic lateral ankle instability have been reported. These techniques can be classified into two basic categories: anatomical reconstruction ${ }^{2-8}$ and tenodesis stabilization. ${ }^{9-13}$ Anatomical reconstruction includes midsubstance imbrication and suturing of the ruptured ligament ends, ${ }^{2}$ augmentation using the extensor retinaculum, ${ }^{3}$ and shortening or reattachment of the ligament using drill holes in the fibula. ${ }^{4-8}$ Anatomical reconstruction is an excellent treatment option because it is simple to perform and complications are rare.

However, if the remnants of the ligaments are significantly damaged, anatomical reconstructions using these remnants can be difficult, and tenodesis stabilization may be required. ${ }^{16}$ In our previously reported series of patients who underwent surgical treatment for chronic lateral ankle instability, $45 \%$ of the cases had severe injuries of either the anterior talofibular ligament or the calcaneo- 
fibular ligament. The results of that study indicated that a talar tilt angle of $\geq 15^{\circ}$ is almost always associated with ligamentous degeneration to scar tissue, rupturing at the endpoints, and/or disappearance $(28 / 36,77.8 \%) .{ }^{17}$ If the remnant consists of scar tissue or is completely absent, anatomical reconstruction may be difficult to perform and tenodesis stabilization is instead indicated.

Several clinical and biomechanical studies have shown that tenodesis stabilization often restricts the ankle ROM and can result in nerve injuries. ${ }^{18-20}$ Tenodesis repair may restrict the ankle ROM if the reconstructed ligament is not located in the original anatomical position. To overcome this problem, we installed reconstructed ligaments in the original anatomical position by devising a system for positioning the drill holes in the bones. As a result, the procedure carried out in the current study did not restrict the ankle ROM.

Sural nerve injuries are often reported as a complication of tenodesis stabilization. ${ }^{21}$ Therefore, the sural nerve was identified and carefully exposed to its distal portion to ensure its protection during the procedure. In the present study, one patient experienced irritation in the sural nerve region; however, no severe complications occurred.

Another drawback of the reported procedure is a residual eversion weakness caused by the sacrifice of the peroneus brevis tendon. We investigated eversion strength after the procedure. Fifteen patients who underwent the operation were included in the study. The eversion strengths of both ankles were measured at the same time using a unique device with a strain gauge. The eversion strength of the operated ankle was similar to that of the non-operated ankle at 13 months after the operation. ${ }^{22}$ In recent studies, patients who underwent a split peroneus brevis tendon reconstruction for lateral ankle instability did well postoperatively from both a functional and patient satisfaction standpoint after 54.8 months of follow up $^{23}$ and after 15 years of follow up..$^{20}$

Two major limitations of the current study include its retrospective nature and the absence of a control group. Furthermore, the complicated maneuvers that are required to perform the surgery and the absence of a longer follow-up period are limitations. Therefore, our procedure should be indicated only for patients with severely damaged ligaments and a talar tilt angle of greater than $15^{\circ}$.

In conclusion, patients treated with lateral ligament reconstruction using a free split peroneus brevis tendon demonstrated good outcomes after 63.8 months of followup. The most important finding of the present study was that our procedure for positioning the graft anatomically was associated with good outcomes without any ankle ROM restrictions. Therefore, our hypothesis was adopted. If the long-term results of conventional tenodesis stabilizations continue to be unsatisfactory, the importance of positioning the graft anatomically may become more widely recognized.

\section{Acknowledgments}

The authors gratefully acknowledge the help of Dr. S. Inokuchi and Professor F. Katsukawa.

\section{Conflicts of Interest}

The authors declare that no conflicts of interest exist.

\section{References}

1. Harrington KD: Degenerative arthritis of the ankle secondary to long-standing lateral ligament instability. J Bone Joint Surg Am 1979; 61: 354-361. PMID:429402, DOI:10.2106/00004623197961030-00006

2. Broström L: Sprained ankles. VI. Surgical treatment of "chronic" ligament ruptures. Acta Chir Scand 1966; 132: 551-565. PMID:5339635

3. Gould N, Seligson D, Gassman J: Early and late repair of lateral ligament of the ankle. Foot Ankle 1980; 1: 84-89. PMID:7274903, DOI:10.1177/107110078000100206

4. Karlsson J, Bergsten T, Lansinger O, Peterson L: Reconstruction of the lateral ligaments of the ankle for chronic lateral instability. J Bone Joint Surg Am 1988; 70: 581-588. PMID:3356725, DOI:10.2106/00004623-198870040-00015

5. Li X, Killie H, Guerrero P, Busconi BD: Anatomical reconstruction for chronic lateral ankle instability in the high-demand athlete: functional outcomes after the modified Broström repair using suture anchors. Am J Sports Med 2009; 37: 488-494. PMID:19251684, DOI:10.1177/0363546508327541

6. Muijs SP, Dijkstra PD, Bos CF: Clinical outcome after anatomical reconstruction of the lateral ankle ligaments using the Duquennoy technique in chronic lateral instability of the ankle: a longterm follow-up study. J Bone Joint Surg Br 2008; 90-B: 50-56. PMID:18160499, DOI:10.1302/0301-620X.90B1.19210

7. Schmidt R, Benesch S, Friemert B, Herbst A, Claes L, Gerngross $\mathrm{H}$ : Anatomical repair of lateral ligaments in patients with chronic ankle instability. Knee Surg Sports Traumatol Arthrosc 2005; 13: 231-237. PMID:15824935, DOI:10.1007/s00167-004-0562-0

8. Trč T, Handl M, Havlas V: The anterior talo-fibular ligament reconstruction in surgical treatment of chronic lateral ankle instability. Int Orthop 2010; 34: 991-996. PMID:20431880, DOI:10.1007/ s00264-010-0995-7

9. Chrisman OD, Snook GA: Reconstruction of lateral ligament tears of the ankle. An experimental study and clinical evaluation of seven patients treated by a new modification of the Elmslie procedure. J Bone Joint Surg Am 1969; 51: 904-912. PMID:4978936, DOI:10.2106/00004623-196951050-00007

10. Morelli F, Perugia D, Vadalà A, Serlorenzi P, Ferretti A: Modified Watson-Jones technique for chronic lateral ankle instability in athletes: clinical and radiological mid- to long-term follow-up. Foot Ankle Surg 2011; 17: 247-251. PMID:22017895, DOI:10.1016/j.fas.2010.08.006

11. Schepers T, Vogels LM, Van Lieshout EM: Hemi-Castaing ligamentoplasty for the treatment of chronic lateral ankle instability: a retrospective assessment of outcome. Int Orthop 2011; 35: 1805-1812. PMID:21637958, DOI:10.1007/s00264-011-1284-9

12. Westlin NE, Vogler HW, Albertsson MP, Arvidsson T, Montgomery F: Treatment of lateral ankle instability with transfer of the extensor digitorum brevis muscle. J Foot Ankle Surg 2003; 42: 183-192. PMID:12907928, DOI:10.1016/S1067-2516(03)70027-1 
13. Yang J Jr, Morscher MA, Weiner DS: Modified Chrisman-Snook repair for the treatment of chronic ankle ligamentous instability in children and adolescents. J Child Orthop 2010; 4: 561-570. PMID:22132034, DOI:10.1007/s11832-010-0299-x

14. Kitaoka HB, Alexander IJ, Adelaar RS, Nunley JA, Myerson MS, Sanders M: Clinical rating systems for the ankle-hindfoot, midfoot, hallux, and lesser toes. Foot Ankle Int 1994; 15: 349-353. PMID:7951968, DOI:10.1177/107110079401500701

15. Becker HP, Ebner S, Ebner D, Benesch S, Frössler H, Hayes A, Gritze G, Rosenbaum D: 12-year outcome after modified Watson-Jones tenodesis for ankle instability. Clin Orthop Relat Res 1999; 358: 194-204. PMID:9973992, DOI:10.1097/00003086199901000-00024

16. Coughlin MJ, Schenck RC, Grebing BR, Treme G: Comprehensive reconstruction of the lateral ankle for chronic instability using a free gracilis graft. Foot Ankle Int 2004; 25: 231-241. PMID:15132931, DOI:10.1177/107110070402500407

17. Hashimoto T, Inokuchi S, Kokubo T: Clinical study of chronic lateral ankle instability: injured ligaments compared with stress Xray examination. J Orthop Sci 2009; 14: 699-703. PMID:19997815, DOI:10.1007/s00776-009-1386-z

18. Rosenbaum D, Engelhardt M, Becker HP, Claes L, Gerngroß H: Clinical and functional outcome after anatomic and nonanatomic ankle ligament reconstruction: Evans tenodesis versus periosteal flap. Foot Ankle Int 1999; 20: 636-639. PMID:10540994, DOI:10.1177/107110079902001004
19. Schmidt R, Cordier E, Bertsch C, Eils E, Neller S, Benesch S, Herbst A, Rosenbaum D, Claes L: Reconstruction of the lateral ligaments: do the anatomical procedures restore physiologic ankle kinematics? Foot Ankle Int 2004; 25: 31-36. PMID:14768962, DOI:10.1177/107110070402500107

20. Ventura A, Legnani C, Corradini C, Borgo E: Lateral ligament reconstruction and augmented direct anatomical repair restore ligament laxity in patients suffering from chronic ankle instability up to 15 years from surgery. Knee Surg Sports Traumatol Arthrosc 2020; 28: 202-207. PMID:30377717, DOI:10.1007/s00167-018$5244-4$

21. Hennrikus WL, Mapes RC, Lyons PM, Lapoint JM: Outcomes of the Chrisman-Snook and modified-Broström procedures for chronic lateral ankle instability. A prospective, randomized comparison. Am J Sports Med 1996; 24: 400-404. PMID:8827297, DOI: $10.1177 / 036354659602400402$

22. Ando C, Kato T, Hosokawa M, Yokoi A, Hashimoto T: Evaluation of the peroneus muscle power after reconstruction of the lateral ligaments of the ankle. J Jpn Soc Surg Foot 1988; 9: 8-11 (In Japanese).

23. Ramdass RS, Grierson KR: A comparison of split peroneus brevis tendon and semitendinosus allograft tendon for lateral ankle ligament reconstruction. J Foot Ankle Surg 2019; 58: 1197-1202. PMID:31679672, DOI:10.1053/j.jfas.2019.04.002 